AUTHOR(S): Peter T. Sheehey, Rickey J. Faehl, Ronald C. Kirkpatrick, and Irvin $R$. Lindemuth

SUBMITTED TO.

Proceedings of MegaGauss-VIII, Eighth International Conference on MegaGauss Magnetic Field Generation and Related Topics 


\section{DISCLAIMER}

This report was prepared as an account of work sponsored by an agency of the United States Government. Neither the United States Government nor any agency thereof, nor any of their employees, make any warranty, express or implied, or assumes any legal liability or responsibility for the accuracy, completeness, or usefulness of any information, apparatus, product, or process disclosed, or represents that its use would not infringe privately owned rights. Reference herein to any specific commercial product, process, or service by trade name, trademark, manufacturer, or otherwise does not necessarily constitute or imply its endorsement, recommendation, or favoring by the United States Government or any agency thereof. The views and opinions of authors expressed herein do not necessarily state or reflect those of the United States Government or any agency thereof. 


\section{DISCLAIMER}

Portions of this document may be illegible in electronic image products. Images are produced from the best available original document. 


\title{
Modeling of Present and Proposed Magnetized Target Fusion Experiments
}

\author{
$P$. Sheehey, R. Faehl, R. Kirkpatrick, and I. Lindemuth \\ Los Alamos National Laboratory \\ Los Alamos, NM 87545 USA
}

\begin{abstract}
In the concept known as Magnetized Target Fusion (MTF) in the United States and Magnitnoye Obzhatiye (MAGO) in Russia, a preheated and magnetized target plasma is hydrodynamically compressed to fusion conditions. Because the magnetic field suppresses losses by electron thermal conduction in the fuel during the target implosion heating process, the compression may be over a much longer time scale than in traditional inertial confinement fusion. Hence "lineron-plasma" compressions, magnetically driven using relatively inexpensive electrical pulsed power, may be practical. One candidate target plasma known as "MAGO" was originated in Russia and is now being jointly developed by the All-Russian Scientific Research Institute of Experimental Physics (VNIIEF) and Los Alamos National Laboratory (LANL). Other possible target plasmas now under investigation at LANL include wall-supported deuterium-fiber-initiated Z-pinches and compact toroids. Detailed computational modeling is being done of such target plasmas. In addition, liner-on-plasma compressions of such target plasmas to fusion conditions are being computationally modeled, and experimental and computational investigation of liner implosions suitable for MTF is continuing. Results will be presented.
\end{abstract}

\section{Introduction}

Magnetized Target Fusion is an approach to controlled fusion that is intermediate between magnetic confinement and inertial confinement fusion (ICF) in time and density scales. Bigger targets and much lower initial target densities than in ICF can be used, reducing radiative energy losses. Reduced losses permit near-adiabatic compression of the fuel to ignition temperatures, even at low (e.g., $1 \mathrm{~cm} / \mu \mathrm{sec}$ ) implosion velocities. In MTF, the convergence ratio $\left(r_{\text {initial }} / r_{\text {final }}\right)$ of the pusher in quasi-spherical geometries may potentially be less than 10 , depending upon the initial temperature of the fuel and the adiabaticity of the implosion. Therefore, "liner-on-plasma" compressions, magnetically driven using relatively inexpensive electrical pulsed power, may be practical [1-4].

An MTF system requires two elements: (1) a preheated and magnetized initial "target" plasma; (2) a target implosion driver. Because the reduced energy losses in MTF relax the power and intensity requirements for an implosion driver, an optimal driver source for MTF might be relatively inexpensive electrical pulsed power, to drive a lineron-plasma implosion. This could utilize either fixed pulsed-power 
facilities, such as Los Alamos' Pegasus or Atlas, or explosive-fluxcompression generators, such as Los Alamos' Procyon or the Russian 200 -MJ-class disk flux compression generators $[3,4]$. Such energy-rich sources might allow a demonstration of fusion ignition via MTF, without a major capital investment in driver technology. The success of magnetized target fusion will depend upon a number of issues. The initial target plasma must meet minimum temperature $(\sim 50 \mathrm{eV}$, preferably $100-$ $300 \mathrm{eV}$ ), density (between $10^{-3}$ and $10^{-6} \mathrm{~g} / \mathrm{cm}^{3}$ ), and magnetic field ( $>50$ $\mathrm{kG}$ ) requirements, and must have a lifetime, adjacent to the supporting wall, greater than the implosion time (typically several $\mu$ sec for a pulsedpower-driven implosion). Plasma-wall interaction must not create dynamical effects or excessive introduction of impurities, which might lead to rapid cooling of the plasma. The target plasma must be readily integrable with drivers for compression to fusion conditions. As it implodes, the liner must remain sufficiently intact that it can effectively compress the target plasma.

LANL and VNIIEF have pursued MAGO/MTF research in recent years, as reported at this conference [3-8] and elsewhere $[9,10]$. Los Alamos is presently investigating three candidate target plasmas: the Russian-originated, explosive-pulsed-power-driven MAGO plasma formation scheme, the high-density Z-pinch, and the Field Reversed Configuration (FRC), an elongated compact toroid. MAGO work includes ongoing joint US-Russian experiment and theory aimed at determining the suitability of the plasma created for MTF compression. A partially wall-supported deuterium-fiber-initiated Z-pinch experiment at LANL has been investigated for MTF applications. Los Alamos is now beginning experimental and theoretical investigation of an FRC plasma for MTF compression. Related experimental and computational work aims to evaluate explosive-flux-compression generators and existing pulsed power facilities as MTF liner drivers. Liner-on-plasma compressions of such target plasmas are being computationally modeled.

\section{MAGO Target Plasmas}

The Russian-originated MAGO plasma formation experiments are the subject of a US-Russian collaboration involving LANL and VNIIEF $[5-7,9,10]$. MAGO is a unique discharge in two cylindrical chambers joined by an annular nozzle. Los Alamos 2-D resistive magnetohydrodynamic (MHD) computations show substantial agreement with the early dynamics of the discharge as evidenced by inductive probes, plasma interferometry, and neutron pulse timing. For times later than about $4 \mu \mathrm{sec}$, the computations predict a relatively stable and uniform wall-confined Z-pinch plasma in the right-hand section of the chamber, with parameters such as density, temperature, and magnetic field very well suited to an MTF target plasma (e.g. $10^{18} \mathrm{~cm}^{-3}, 100-300$ $\mathrm{eV}, 200 \mathrm{kG}$ ). However, if the plasma becomes contaminated by wall or insulator constituents, enhanced radiative cooling and mass dilution could drop the high late-time plasma temperatures rapidly. Hence, 
measurement of late-time temperature and composition of the MAGO plasma is a major focus of continuing experiments. Recently, the International Science and Technology Center has supported MAGO experiments in smaller, 6-cm-radius chambers. Los Alamos simulations predict that $100 \mathrm{eV}$ temperatures could be produced in such discharges with $\mathrm{O}(4 \mathrm{MA})$ peak currents, but that a recent joint LANL-VNIIEF shot with $2.5 \mathrm{MA}$ peak current probably reached less than $50 \mathrm{eV}$.

\section{Fiber-Initiated Z-Pinch Target Plasmas}

Another possible magnetized target plasma configuration, on which there is extensive experience at Los Alamos National Laboratory, is the deuterium-fiber-initiated Z-pinch [7,10]. 2-D MHD modeling of a fiber Z-pinch plasma contained inside a $2-\mathrm{cm}$-radius conducting wall predicts that, after an instability-driven expansion to the wall, the plasma settles into a dense, hot, Kadomtsev-stable state, also with the desired MTF target plasma parameters. An experiment was built to investigate such plasmas, but deuterium fiber shots have not been executed due to problems with producing and inserting a deuterium fiber. Polyethylene fiber shots have been done, but simulations of polyethylene-fiberinitiated plasmas predict that, although similar densities and temperatures may be produced, the interesting Kadomtsev-stable state may not occur. Further effects not yet modeled include three-dimensional effects and plasma-wall material interactions. Late-time MAGO plasmas and deuterium-fiber plasmas appear similar in some respects: similarly stable pinches (although the MAGO plasma truncates the inner portion of the pinch with its central conductor), both involving wall support of plasma.

\section{FRC Target Plasmas}

A new effort at Los Alamos aims to develop Field Reversed Configuration (FRC) plasmas for MTF targets. One potential advantage of such plasmas over MAGO/Z-pinch direct-current-coupled plasmas is that FRCs, once formed, have current decoupled from the walls, removing that potential source of contaminants; FRCs can also be translated from a formation chamber to a liner compression chamber. Plans are to start with an FRC in the $10^{17} \mathrm{~cm}^{-3}$ density range, which is on the low end of that desired for MTF. An FRC target plasma experiment is being designed and modeled at Los Alamos, and initial liner compression experiments designed for an FRC are to take place on the Shiva-Star pulsed power facility at the Air Force Research Laboratory.

\section{High-Energy Liners and Liner-on-Plasma Compression}

A joint LANL-VNIIEF experiment (high energy liner "HEL-1") executed in August, 1996, put approximately $100 \mathrm{MA}$ through a $24-\mathrm{cm}-$ radius, 4-mm-thick, $10-\mathrm{cm}$-long aluminum cylindrical liner, which, by the time it reached the $5.5 \mathrm{~cm}$ radius of the central measuring (diagnostic) unit (CMU), was imploding at $7 \mathrm{~mm} / \mu \mathrm{sec}$ and contained approximately $20 \mathrm{MJ}$ of kinetic energy [7,8]. Such a liner approaches 
the energy and velocity regime required to drive an MTF target such as MAGO to fusion conditions. Los Alamos Eulerian 2-D MHD computations including the effects of material strength, resistive field diffusion, glide-plane-liner interactions, and possible nonlinear instability development, gave results in substantial agreement with experimental measurements. One interesting feature noted is that, although early current loading of the liner starts it out with a substantial inward bowing which might be expected to seed long-wavelength Rayleigh-Taylor instability growth, the ultimate shape of the liner as it meets the CMU is with a pronounced outward bowing, due to strong end effects; mass thinning along the glide planes results in liner "glide plane run-ahead," and magnetic flux penetration along these areas.

Liner-on-plasma MTF experiments employing HEL-1-like explosive pulsed power drivers, or fixed pulsed power facilities such as LANL's Atlas, are now being computationally modeled.

\section{Conclusions}

Magnetized Target Fusion (MTF) is an approach to controlled fusion which potentially avoids the difficulties of the traditional magnetic and inertial confinement appoaches. It appears possible to investigate the critical issues for MTF at low cost, relative to traditional fusion programs, utilizing pulsed power drivers much less expensive than ICF drivers, and plasma configurations much less expensive than those needed for full magnetic confinement. If inexpensive electrical pulsed power could be used as the implosion driver, smaller, more economically viable fusion reactors might be possible. Because of MTF's qualitative differences from the inertial or magnetic confinement approaches to fusion, MTF reactors will have different characteristics and trade-offs, enhancing the prospects for practical fusion power. Computational and experimental research into MTF is proceeding at Los Alamos, VNIIEF, and other laboratories.

\section{References}

1. I. Lindemuth and R. Kirkpatrick, Nuclear Fusion 23, 263 (1983).

2. V. Mokhov et al., Sov. Phys. Dokl. 24, 557 (1979).

3. V. Chernyshev et al., in Megagauss Fields and Pulsed Power Systems, ed. V. Titov \& G. Shvetsov, p. 347, Nova Science Publishers, NY(1990). 4. A. Pavlovskii et al., ibid., p. 327.

5. V. Chernyshev et al., in Megagauss Magnetic Field Generation and Pulsed Power Applications, ed. M. Cowan and R. Spielman, Part 1, p. 557, Nova Science Publishers, New York (1995).

6. I. Lindemuth et al., ibid., p. 361.

7. P. Sheehey et al., MegaGauss-VII Proceedings, Sarov, Russia (1996).

8. R. Reinovsky et al., ibid.

9. I. Lindemuth, V. Chernyshev, V. Mokhov et al., Physical Review Letters 75(10), 1953 (1995).

10. P. Sheehey et al., Fusion Technology 30, 1355 (1996). 